\title{
The Pharmacological Treatment Options for Pediatric Migraine: An Evidence-Based Appraisal
}

\author{
Donald W. Lewis, MD, Paul Winner, DO \\ Division of Pediatric Neurology, Children's Hospital of the King's Daughters, Eastern Virginia Medical School, \\ Norfolk, Virgnia 23501
}

Summary: The treatment of children and adolescents who suffer from migraine headaches must be individually tailored, flexible, and balanced with a blend of bio-behavioral measures, agents for acute treatment and, if needed, daily preventive medicines. While controlled data is limited, there is now enough evidence available to provide a rational framework to build treatment plans appropriate for the pediatric population. Essentially, the pharmacological management of pediatric migraine divides into agents for the acute attacks and agents used daily to prevent or reduce the frequency of attacks. For the acute treatment, the most rigor- ously studied agents are ibuprofen, acetaminophen, and the nasal spray forms of sumatriptan and zolmitriptan, all of which have shown both safety and efficacy in controlled trials. For preventive treatment the calcium channel blocker flunarezine has the best efficacy profile in controlled trials, but is not available in the U.S. A growing body of data, mostly uncontrolled, is emerging regarding the use of several anti-epileptic agents (e.g. topiramate, disodium valproate, levateracetam), as well as the antihistamine cyproheptadine and the anti-depressant amitriptyline. Key Words: Migraine, treatment, children, adolescents.

\section{INTRODUCTION}

Migraine headaches represent a recurring pattern of attacks of intense, throbbing, nauseating frontal or temporal head pain which typically last for hours, but may last for 2 or 3 days. Though well recognized in adults, migraines are quite common in children, even young children but often go unrecognized or misattributed to causes such as refractive errors, sinus disease or attention getting behaviors. Excellent epidemiological surveys have demonstrated that the prevalence of migraine headache steadily rises through childhood and a curious, unexplained, gender shift occurs (table 1). For boys, the mean age of onset of migraine is 7 years for boys and 10.9 years for girls. ${ }^{1}$

\section{DIAGNOSTIC CRITERIA}

What features of a recurring headache syndrome in a child distinguish the attacks as being due to $m i$ graine?

Division of Pediatric Neurology, Children's Hospital of the King's Daughters, Eastern Virginia Medical School, Norfolk, Va, Telephone: 7576689920 Fax:757 6689930 Email:dlewis@chkd.com
The first attempts to define migraine as a clinical entity in children began in the early 1950's. Through the next two decades, several proposals were introduced with the common features including episodic, paroxysmal headache separated by pain free intervals, accompanied variable number of associated features including visual aura, nausea, abdominal pain, throbbing quality, unilateral location of pain, and family history of migraine. ${ }^{2,3}$

The currently accepted criteria for the classification and diagnostic criteria for Pediatric Migraine were published by the International Headache Society (I.H.S.) in 2004 and represent a thoughtful, developmentally sensitive, and clinically relevant consensus which maintain the specificity necessary to conduct clinical trials and improve sensitivity to permit their clinical utility in pediatric practice. ${ }^{4}$ The currently accepted classification system for migraine is shown in table 2 and the diagnostic criteria for migraine without aura are shown in table 3 .

\section{MANAGEMENT OF PEDIATRIC MIGRAINE}

The goals for migraine management as stated in an American Academy of Neurology Practice Parameter (www.aan.org) are to: 
TABLE 1. The prevalence of migraine headache through childhood

\begin{tabular}{cccl}
\hline \multicolumn{1}{c}{ By age } & Preschool & $\begin{array}{c}\text { Elementary } \\
\text { School }\end{array}$ & $\begin{array}{c}\text { High } \\
\text { School }\end{array}$ \\
\hline $\begin{array}{l}\text { Prevalence: } \\
\text { Gender ratio: }\end{array}$ & $\begin{array}{c}1.2-3.2 \% \\
\text { boys }>\text { girls }\end{array}$ & $\begin{array}{l}4-11 \% \\
\text { boys }=\text { girls }\end{array}$ & $\begin{array}{l}8-23 \% \\
\text { girls }>\text { boys }\end{array}$ \\
\hline
\end{tabular}

1. reduction of headache frequency, severity, duration, and disability;

2. reduction of reliance on poorly tolerated, ineffective, or unwanted acute pharmacotherapies;

3. improvement in the quality of life;

4. avoidance of acute headache medication escalation;

5. education and enablement of patients to manage their disease to enhance personal control of their migraine; and

6. reduction of headache-related distress and psychological symptoms. ${ }^{5}$

While these goals were developed for migraine in adults, they are equally valid for the pediatric population.

In order to achieve the goals, an individually tailored, flexible, and balanced blend of bio-behavioral measures, pharmacological agents for acute treatment and, if needed, daily preventive medicines may be considered. The treatment options may be divided into three catagories:

TABLE 2. 2004 International Classification of Headache Disorders (ICHD) for Migraine

Migraine
Migraine without aura
Migraine with aura
Typical aura with migraine headache
Typical aura with non-migraine headache
Typical aura without headache
Familial hemiplegic migraine
Sporadic hemiplegic migraine
Basilar-type migraine
Childhood periodic syndromes that are commonly
precursors of migraine
Cyclical vomiting
Abdominal migraine
Benign paroxysmal vertigo of childhood
Retinal migraine
Complications of migraine
Chronic migraine
Status migraine
Persistent aura without infarction
Migrainous infarction
Probable migraine

(1) bio-behavioral strategies (e.g. life style modifications, stress management, biofeedback)

(2) acute therapies (e.g. analgesics, anti-emetics, "triptan" agents) and

(3) preventive measures (both pharmacological and non-pharmacological).

The initial phase of developing an individualized treatment plan is to appreciate the degree of disability imposed by the patient's headache, referred to as the headache "burden." Understanding of the impact of the headache on the quality of life (e.g. school attendance) will guide in the decisions regarding the most appropriate therapeutic blend. ${ }^{6}$

The next phase is to establish the headache pattern. How often are the headaches occurring? Is there are predictable pattern (e.g. menstrual cycle, school calendar, examination schedules)? In order to appreciate patterns, headache calendars are invaluable to determine the frequency and duration of headache and to help identify precipitating or provocative phenomena. Knowledge of the headache pattern will guide the clinical decisions necessary to tailor the treatment to the patient.

For example, a teenager who documents multiple, prolonged migraine attacks each week during the school year who misses about 2 days of school per month is experiencing significant headache burden and will likely require a blend of management options including bio-behavioral interventions (e.g. biofeedback), acute medicines (e.g. nasal sumatriptan or zolmitriptan spray) and a daily preventative agent (e.g. amitriptyline, topiramate, or valproic acid) during the school year.

Alternatively, an 8 year old with one or two 1 hour attacks each month of frontal, pounding, nauseating headache at school starting after lunch would require a less aggressive program. Since the headache calendar documents that the attacks occur after lunch, at school, the question would arise regarding what is happening at lunchtime? Is there a bully in the cafeteria or is it the loud noise and chaos of the lunchroom precipitating the headache? Might this child benefit from eating their lunch in the quiet and safety of the library or in a classroom? In this case description, if medications are necessary, a simple analgesic agent such as acetaminophen $(15 \mathrm{mg} / \mathrm{kg}$ or ibuprofen 7.5 $10 \mathrm{mg} / \mathrm{kg}$ ) should be readily available to the child at school and the patient must be educated to request the medicine as soon as the headache begins. This patient likely requires no daily medications.

A complete understanding of the headache burden must be appreciated before a rational, comprehensive treatment regimen can be instituted. 
TABLE 3. 2004 International Headache Society Criteria for Pediatric Migraine without Aura Diagnostic Criteria

A. At least 5 attacks fulfilling criteria B-D (below)

B. Headache attacks lasting 1-72 hours

C. Headache has at least two of the following characteristics:

1. Unilateral location, may be bilateral, frontotemporal (not occipital)

2. Pulsing quality

3. Moderate or severe pain intensity

4. Aggravation by or causing avoidance of routine physical activity (e.g. walking or climbing stairs)

D. During the headache, at least one of the following:

1. Nausea and/or vomiting

2. Photophobia and phonophobia, which may be inferred from their behavior

E. Not attributed to another disorder

\section{Bio-behavioral strategies}

While all children and adolescents with migraine will benefit from a review of the basic bio-behavioral measures including sleep hygiene, exercise, dietary modifications, biofeedback, and stress management, this review will focus on the pharmacological options. Omission of discussion of these strategies does not diminish their clinical value.

\section{Acute treatments}

Treatment of an acute migraine attack may include an analgesic, an anti-emetic or a specific anti-migraine agent (e.g. "triptan"). Anagesics represents the mainstay of migraine management in children. Although a wide variety of medicines are commonly used in the acute treatment of migraine, none have a defined indication for use in children from the U.S. FDA (table 4). A 2004 American Academy of Neurology Practice Parameter focusing on the treatments of migraine in children and adolescents provides an evidence-based review and series of recommendations regarding treatment options (www.aan.org). ${ }^{1}$
There are several guiding principles of acute treatment which must be incorporated into the education process:

(1) Take the medicine as soon as possible after the headache begins. This principle mandates that the treatment (e.g. tablet, nasal spray) must be available to the patient where their headaches usually occurs (e.g. school);

(2) Take the appropriate dose (e.g. $7.5-10 \mathrm{mg} / \mathrm{kg}$ of ibuprofen);

(3) Seek a quiet, dark place to rest following ingestion of medicine;

(4) Avoid analgesic overuse.

\section{Analgesics}

Simple analgesics and non-steroidal anti-inflammatory drugs (NSAIDs) are usually quite effective in children and adolescents with mild to moderate migraine.

Ibuprofen $(7.5-10 \mathrm{mg} / \mathrm{kg})$ has been shown to be safe and effective in the treatment of childhood migraine in

TABLE 4. Acute Treatment for Childhood Migraine

\begin{tabular}{|c|c|c|}
\hline Drug & Dose & Available \\
\hline Acetaminophen\# & $10-15 \mathrm{mg} / \mathrm{kg} / \mathrm{dose}$ & tabs $80,160,325 \mathrm{mg}$ \\
\hline Ibuprofen \# & $10 \mathrm{mg} / \mathrm{kg} / \mathrm{dose}$ & $\begin{array}{l}\text { chewable tabs } 100 \\
\text { syrup } 100 \mathrm{mg} / \mathrm{tsp} \\
\text { tabs } 200,400,600,800\end{array}$ \\
\hline $\begin{array}{l}\text { Naproxen sodium } \\
\text { 5-HT agonist: }\end{array}$ & $2.5-5 \mathrm{mg} / \mathrm{kg}$ & tab $220,250,375,500 \mathrm{mg}$ \\
\hline \multicolumn{3}{|l|}{ Sumatriptan* } \\
\hline Tablet & & $25 \mathrm{mg}, 50 \mathrm{mg}, 100 \mathrm{mg}$ \\
\hline Subq injection & & $6 \mathrm{mg}$ \\
\hline Nasal spray \# & & $5 \mathrm{mg}, 20 \mathrm{mg}$ \\
\hline Zolmitriptan* & & $2.5 \mathrm{mg}, 5 \mathrm{mg}$ \\
\hline Tablet & & $2.5 \mathrm{mg}, 5 \mathrm{mg}$ \\
\hline Oral disintegrating tablet & $2.5 \mathrm{mg}, 5 \mathrm{mg}$ & \\
\hline Nasal spray \# & & $5 \mathrm{mg}$ nasal spray \\
\hline \multicolumn{3}{|l|}{ Rizatriptan* } \\
\hline Tablet & & $5,10 \mathrm{mg}$ \\
\hline Oral disintegrating tablet & & $5,10 \mathrm{mg}$ \\
\hline
\end{tabular}

\# = Efficacy and safety data in adolescents (12-17 years of age) $*=$ not approved for pediatric use 
two double-blind, placebo-controlled trials. The first study ( $\mathrm{n}=88)$ compared ibuprofen $(10 \mathrm{mg} / \mathrm{kg})$ to acetaminophen $(15 \mathrm{mg} / \mathrm{kg})$ and to placebo. At the one hour and two hour endpoints, both ibuprofen and acetaminophen were significantly more effective than placebo in providing "pain relief" as defined by a 2 point reduction of pain on a 5 point pain scale $(\mathrm{p}<0.05)$. At the 2 hour endpoint, ibuprofen provided "alleviation" of headache in $56 \%$ of treated patients compared $53 \%$ for acetaminophen and $36 \%$ for the placebo group. Differences between ibuprofen compared to acetaminophen were not statistically significant at the 2 hours endpoint. Complete resolution of headache was found in $60 \%$ of ibuprofen treated children, $39 \%$ of the acetaminophen group versus $28 \%$ of those who received placebo. Reduction in moderate to severe headache by at least two grades after two hours was twice as likely with acetaminophen and three times as likely with ibuprofen as placebo. Acetaminophen was considered effective and well tolerated. Acetaminophen was observed to have a faster onset of action than ibuprofen. ${ }^{7}$

The second study of ibuprofen $(7.5 \mathrm{mg} / \mathrm{kg})$ included 84 children ages 6-12 years and found that there was a significant reduction in headache severity (4 point pain scale) in $76 \%$ of those on active drug versus $53 \%$ in the placebo group at the primary two hour end point $(\mathrm{p}<0.01)$. Reduction in pain score, absence of nausea, and reduced need for rescue medications all reached statistical significance $(\mathrm{p}<0.05)$. An unexplained observation in this study was the differential response between boys and girls. The boy's 2 hour headache response was $84 \%$ with ibuprofen versus $43 \%$ with placebo $(\mathrm{p}<0.01)$, whereas the girl's response rate were $65 \%$ with ibuprofen and $67 \%$ with placebo (NS). ${ }^{8}$

There is no data to support or refute the role of other NSAIDs in pediatric migraine (e.g. naproxen, ketorolac).

Combination agents (e.g. aspirin, acetaminophen, caffeine, butalbital) have not been studied in children. Aspirin containing preparations should not be used in children who proximate to febrile illness because of concerns regarding a relationship to Reye Syndrome.

Overuse of "over-the-counter" analgesics can be an aggravating phenomenon. Recognized in adult populations years ago, overuse (e.g. $>5$ times / week) of acetaminophen, ibuprofen, and, to a lesser extent, aspirincontaining compounds can be a contributing factor to frequent, even daily, headache patterns. When recognized, patients who are overusing analgesics must be educated to discontinue the practice.

\section{Triptans}

The introduction of the 5-hydroxytryptamine (5-HT1) agonists has revolutionized the treatment of moderate to severe migraine attacks in adults. The 5-HT1 agonists have been studied in children and adolescents in both open label and double blind studies, but the results have yet to convincingly demonstrate their efficacy, thus none are currently approved by the U.S. FDA.

Sumatriptan. Sumatriptan has been the most rigorously studied "triptan" in adolescents (12-17 years). Available insubcutaneous injection, tablet, and nasal spray, and the tablets and nasal formulations are preferable for use in children.

Sumatriptan in the tablet form $(50-100 \mathrm{mg})$ was studied in one clinical trial including children aged 8.3 -16.4 years $(n=23)$, but failed to clearly demonstrate efficacy greater than matched placebo at the primary endpoint of pain relief at 2 hours (difference 9\%, 95\% CI for difference -21 to $38 \%, \mathrm{p}=\mathrm{NS}){ }^{9}$ Another adolescent study, a multi-centered, double-blind, placebo-controlled trial of $25 \mathrm{mg}, 50 \mathrm{mg}$, and $100-\mathrm{mg}$ tablets $(n=302)$ failed to demonstrate efficacy greater than placebo at the 2 hour primary endpoint, but secondary endpoints were found statistically significance with doses of 25,50 , and $100 \mathrm{mg}$ at the 3 and 4 hour endpoints showing $74 \%$ pain relief at the 4-hour mark. $^{10}$

Common side effects included warmth, tightness, burning, stinging, numbness, strangeness, and pressure occurred in 0 to $1 \%$ at the $25-\mathrm{mg}$ dose, compared to 1 to $4 \%$ of adolescents at the 50 and $100 \mathrm{mg}$ doses. The side effects of chest discomfort, tightness, pressure and heaviness ranged from 0 to $1 \%$ for the $25-\mathrm{mg}$ tablets as compared with 0 to $3 \%$ for the placebo and 3 to $4 \%$ for the 50-mg tablets.

No serious adverse events were documented in this study of 302 patients. Cardiovascular palpitations, tachyarrhythmias, and hypotension were reported to be 0 to $1 \%$ for the 25 -and 50 -mg tablets and slightly higher for the 100-mg tablets. No such side effects were reported in the placebo group.

Sumatriptan nasal spray was studied in a randomized, double-blind, placebo-controlled trial in which adolescents ages $12-17$ years were treated for a single attack with three nasal spray doses: 5,10 , and $20 \mathrm{mg}$. At 1 hour, $56 \%$ of patients using the 10 - or $20-\mathrm{mg}$ dose of sumatriptan nasal spray reported significant headache relief compared with $41 \%$ in the placebo group (2 point reduction in pain on using a 4 point pain scale). All three doses were superior to the placebo with respect to the cumulative percentage of patients who obtained headache relief within 2 hours of administration. When reviewing the pain free data, the $20 \mathrm{mg}$ sumatriptan nasal spray provided a statistically significant greater response of $36 \%$ at 2 hours compared with the placebo at $25 \%(p<.05){ }^{11}$

Photophobia was significantly lower with the $20-\mathrm{mg}$ 
sumatriptan nasal spray at 2 hours compared with the placebo. There were no differences noted in the rescue medication of the second dose of sumatriptan in either the sumatriptan or placebo groups. There was no difference in headache recurrence among the three treatment groups, which ranged from 16 to $20 \%$, and the placebo group at $20 \%$. Headache recurrence was lower in the 10 and $20-\mathrm{mg}$ sumatriptan nasal spray patient groups at 8 hours and 8.2 hours respectively as compared with the placebo group at 6.7 hours. ${ }^{11}$

The most common adverse event reported by this patient group was taste disturbance. No serious adverse event was reported in the sumatriptan treatment population in this study.

A recently published report pooled efficacy and tolerability results of $5 \mathrm{mg}$ and $20 \mathrm{mg}$ sumatriptan nasal spray versus placebo from two large US randomized, placebo-controlled, double-blind, parallel-group studies in adolescents 12 to 17 years of age with at least 6-month history of migraine. The composite population included 1105 patients (placebo $\mathrm{n}=374 ; 5 \mathrm{mg}$ $\mathrm{n}=377 ; 20 \mathrm{mg} \mathrm{n}=354$ ). Subjects in both trials treated a single moderate or severe migraine attack. The percentage of subjects with headache relief (moderate/ severe pain reduced to mild or none) or pain free (moderate/severe pain reduced to no pain) was evaluated at $30 \mathrm{~min}, 1$ hour, and 2 hours. Sustained pain relief (i.e. headache relief maintained without recurrence or need for rescue medication) from within 24 hours was also analyzed. Sumatriptan nasal spray $20 \mathrm{mg}$ demonstrated significantly greater headache relief compared to placebo at $30 \mathrm{~min}(39 \%$ vs. $30 \%$, $\mathrm{p}=0.016), 1$ hour $(59 \%$ vs. $48 \%, \mathrm{p}=0.007)$, and 2 hours $(67 \%$ vs. $57, \mathrm{p}=0.005)$. Sumatriptan nasal spray $5 \mathrm{mg}$ showed significantly greater headache relief compared to placebo at 2 hours $(64 \%$ vs. $57 \%, p=0.037)$. Sustained relief was greater for sumatriptan nasal spray $5 \mathrm{mg}(37 \%, \mathrm{p}=0.041)$ and for sumatriptan nasal spray $20 \mathrm{mg}(41 \%, \mathrm{p}=0.003)$. The pain free endpoint was reached with sumatriptan nasal spray $20 \mathrm{mg}$ compared to placebo at $1 \mathrm{hr}(20 \%$ vs. $14 \%, \mathrm{p}=0.034)$ and 2 hrs $(42 \%$ vs. $28 \%, p=0.001)(12)$.

The most frequent treatment related adverse events for placebo, $5 \mathrm{mg}$ and $20 \mathrm{mg}$ sumatriptan nasal spray, respectively, were: taste disturbance $(2 \%, 19 \%, 26 \%)$, nausea $(3 \%, 4 \%, 7 \%)$, vomiting $(1 \%, 2 \%, 4 \%)$ and burning /stinging sensation ( $1 \%, 1 \%, 3 \%)$. Infrequent (1\%) were pressure/tightness and chest symptoms reported in each of the treatment groups. ${ }^{12}$

On the basis of these studies, the AAN Practice Parameter stated: "Sumatriptan nasal spray is effective and should be considered for the acute treatment of migraine in adolescents."

From clinical experience with sumatriptan nasal spray in adolescents, the disturbance in taste may be mitigated in many patients by the use of flavored lozenges or hard candy after administration of the nasal spray. The taste disturbance also may be lessened by careful demonstration of the techniques of correct administration of the nasal spray medication.

Subcutaneous sumatriptan. In an open-label study, Linder, documented the effectiveness of subcutaneous sumatriptan $0.06 \mathrm{mg}$ per $\mathrm{kg}$ and showed an overall efficacy of $72 \%$ at 30 minutes and $78 \%$ at 2 hours, with a recurrence rate of $6 \%$. Due to the tendency that children report shorter headache duration, a recurrence rate of $6 \%$ would seem appropriate for this study population. ${ }^{13}$

Rizatriptan. Rizatriptan (Maxalt) 5-mg tablets have been evaluated in patients ages 12 - 17 years in a doubleblind, placebo-controlled, parallel-group, single-attack study. A total of 149 adolescents were treated with rizatriptan $5 \mathrm{mg}$, and 147 were treated with a placebo. The majority of patients used one dose of study medication. The percentage of adolescents receiving pain relief at 2 hours for the rizatriptan 5-mg group was $66 \%$; this is similar to that seen in the adult population receiving a 5-mg dose but is not statistically significant since the response of the placebo group was $57 \%$. The headachefree status at 2 hours was $32 \%$ for the rizatriptan 5-mg group and $28 \%$ for the placebo. There were no serious adverse events. The most common adverse events reported were fatigue, dizziness, somnolence, dry mouth, and nausea. With regard to functional disability, significantly more adolescent patients $(44 \%)$ on rizatriptan 5 $\mathrm{mg}$ had no functional disability at 2 hours as compared with the placebo group (36\%). Rizatriptan $5 \mathrm{mg}$ is well tolerated in this adolescent population study. ${ }^{14}$

Zolmitriptan. Zolmitriptan (Zomig) has been studied in a subgroup of 38 adolescents (12 to 17 years) entered in a one-year open label phase of a large study. The first two migraine subgroups were treated with $2.5 \mathrm{mg}$ and subsequent attacks with 2.5 or $5 \mathrm{mg}$ at each patient's discretion. The overall headache response at 2 hours was $80 \%$ (88\% and $70 \%$ with zolmitriptan, $2.5 \mathrm{mg}$ and $5 \mathrm{mg}$ respectively). Treatment was well tolerated..$^{15}$

Zolmitriptan nasal spray was studied in a multi-center, randomized, double-blind, placebo-controlled, 2 attack crossover trial with a single-blind placebo challenge $(n=171,275$ attacks analyzed). Each attack was initially treated with placebo NS (single-blind). If a headache response was obtained at 15 minutes, no further medication was taken. If headache pain remained moderate or severe, patients then treated with zolmitriptan NS or placebo NS (double-blind). Using the intent to treat (ITT) population, the 1 hour headache response was analyzed for those patients who did not respond to placebo in the first 15 minutes and who treated one or two headaches. The mean age was 14.1 years, $58.5 \%$ female with $62.5 \%$. Zolmitriptan nasal spray produced significantly higher headache response rates than placebo at 
TABLE 5. Preventative Agents for Childhood Migraine

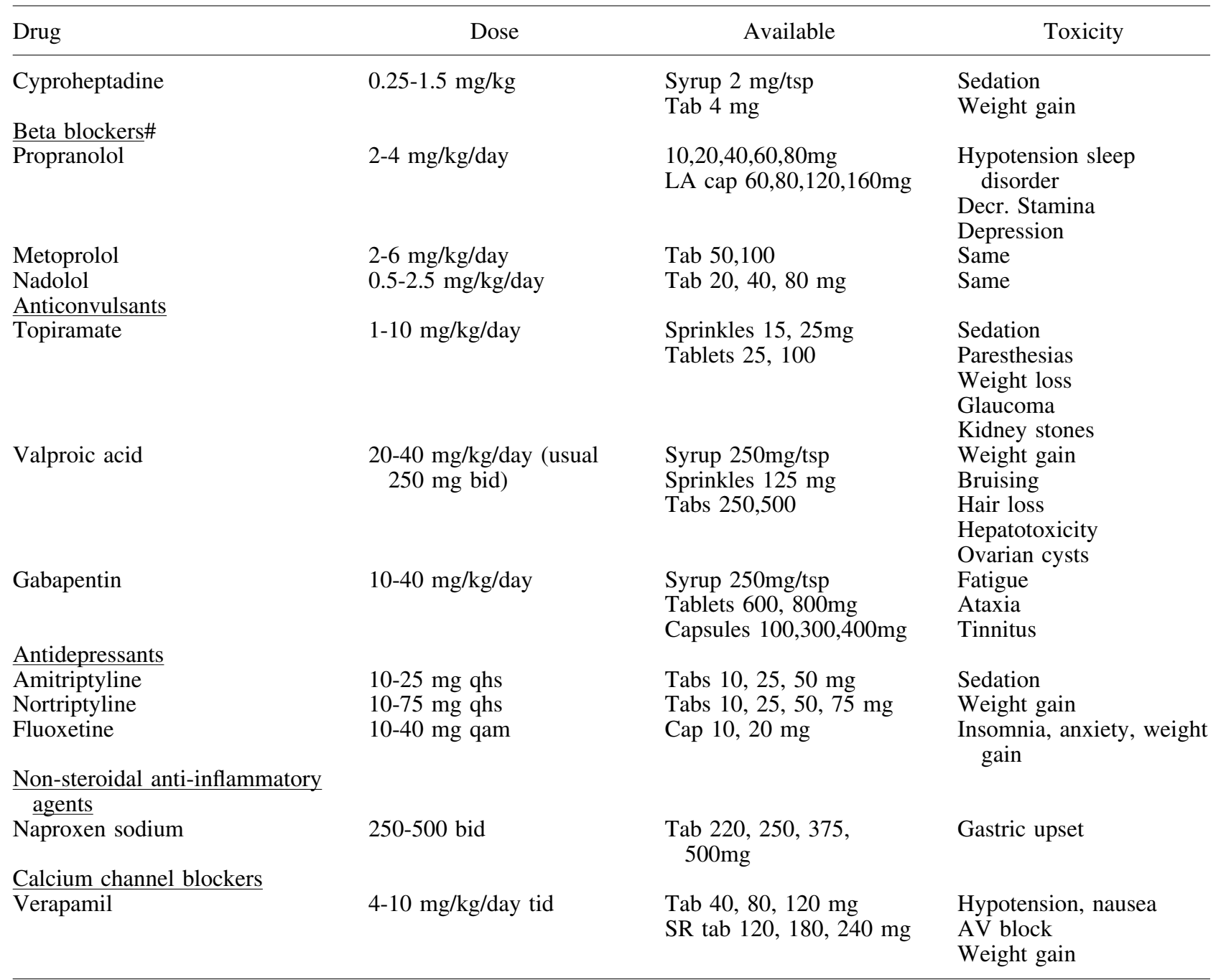

\footnotetext{
\# AVOID WHEN: Asthma, Diabetes
}

1 -hour post-dose ( $58.1 \%$ versus $43.3 \%$; $\mathrm{p}<0.02)$, with an onset of action as early as 15 minutes. The 1 hour pain free rate for zolmitriptan nasal spray was $27.7 \%$ vs $10.2 \%$ for placebo $(\mathrm{p}<0.01)$. The two hour sustained headache response rate for zolmitriptan nasal spray was $53.4 \%$ vs $36.2 \%$ for placebo $(p<.01)$. Fifty one $\%$ of patients treated with zolmitriptan were able to return to normal activities at 1 hour vs $37.5 \%$ treated with placebo $(\mathrm{p}<.03)$. Adolescents experienced a low incidence of adverse events with dysgeusia being the most common $\mathrm{AE}(6.5 \%)$. There were no serious adverse events and no withdrawals due to adverse events. This study concluded that zolmitriptan nasal spray was statistically superior to placebo for the treatment of migraine in adolescents for the one hour headache response in the ITT population and was well tolerated in adolescent patients with migraine, with a fast onset of action. ${ }^{16}$

\section{Strategies for "triptan" use in children and adolescents}

None of the "triptans" are approved for use in children and adolescents by the U.S. FDA, so any suggestions regarding their use must be judged as "off label." The previously presented data does suggest both efficacy and safety, therefore the "triptans" may be considered for use in adolescents who experience severe migraine attacks which have shown to be refractory to simple analgesics, assuming that there are no medical contraindication (table 5). Generally, treatment should be initiated at the low dose, which can then be repeated if there is not adequate response. Subsequent attacks should then be treated with a higher dose to assess if this provides better efficacy. The goal is to obtain a "pain free" status for the child or adolescent within 2 hours; if this has not occurred, the same "triptan" medication may be repeated. 
Although the associated symptoms of migraine such as nausea, photophobia, or phonophobia often respond to triptans, they may not be completely relieved.

The data presented in the above section would suggest that the most efficacious agents for use in adolescents would include the nasal spray forms of sumatriptan and zolmitriptan.

\section{Anti-emetics}

Anti-emetics, available in suppository, sublingual and the oral forms, may be necessary for many children and adolescents with acute migraine attacks which are accompanied by intense nausea or vomiting. Interestingly, simple analgesics such as ibuprofen, when used early in the course of a migraine attacks, provide significant relief from both nausea and vomiting. Specific anti-migraine agents such as the "triptans" generally provide relief from pain as well as other accompanying autonomic symptoms.

Promethazine, prochlorperazine, metoclopromide, and ondansetron, at appropriate doses for the child's weight, can be quite effective in children who have disabling nausea or vomiting with their migraine attacks. Antiemetics may be used effectively in conjunction with other acute therapies (e.g. ibuprofen, naproxen) for treatment of moderate to severe migraine attacks in children and adolescents. The most significant precaution in children is the potential for extrapyramidal side effects (e.g. oculogyric crisis, dystonic reactions).

\section{Preventive measures}

A diverse group of medications are used to prevent frequent attacks of migraine, though few have clearly demonstrated efficacy in controlled trials (table 5). Their use should, however, be limited to those patients whose headaches occur with sufficient frequency or severity as to impose disability and thereby justify a daily treatment regimen. Most clinical trials have required a minimum of 3 headaches per month to justify a daily agent. A clear sense of functional disability must be established before committing to a course of daily medication. It is also useful to identify the presence of "co-morbid conditions" (e.g. anxiety, depression, obesity) which may suggest the relative benefit of one agent over another.

The duration of treatment is also controversial. In recognition of the cyclical nature of migraine, the daily agents should be used for a finite period of time. The general recommendation is to provide treatment through the calendar school year, then gradually eliminate daily agents during summer vacation. Another option in younger children is to use a shorter course (e.g. 6-8 week), followed by a slow withdrawal.

While there is an unfortunate lack of controlled data regarding drug therapies for migraine prophylaxis in children, data is beginning to emerge. Use of the many of these agents listed in Table 5 is based upon anecdotal information or extrapolated adult experiences.

\section{Cyproheptadine (Periactin)}

This anti-histamine with both anti-serotonergic and calcium channel blocker properties has not been subjected to controlled trials, but is widely used for migraine prevention in young children (generally $<12$ years of age).

A single retrospective study of the use of preventative agents for children and adolescents within one child neurology practice found that headache frequency was reduced from a mean baseline of 8.4 headaches/month to 3.7 headaches per month. A positive response rate, defined as an overall favorable decrease in headache frequency and intensity plus acceptability of the agent, was noted in $83 \%(n=30)$. Common side effects included sedation and increased appetite. ${ }^{17}$

Dosing schedules vary widely from single bedtime schedules to tid regimens. A dose of 2-4 mg orally at bedtime is a rational starting point with option to increase to a maximum of 12-16 mg per day divided tid.

Cyproheptadine has two major limiting features: sedation and appetite stimulation. These effects restrict its acceptability in adolescence, but may be advantageous in thin pre-adolescents.

\section{Beta-blockers}

Propranolol has been studied in 3 randomized, doubleblind studies, but the results have failed to consistently demonstrate effectiveness. Nonetheless, beta-blockers are often viewed as one of the first line agents in childhood.

The first study was a double-blind, crossover trial in children ages $7-16$ years $(n=28)$ using $60-120 \mathrm{mg}$ per day $(0.5-1 \mathrm{mg} / \mathrm{kg} /$ day divided tid $)$. Among the propranolol treated patients, 20 of 28 patients $(71 \%)$ had complete remission from headaches and another 3 patients (10\%) experience a $66 \%$ reduction in headache frequency. In the placebo group, $3 / 28$ had complete remission and 1 of the 28 experienced a $66 \%$ improvement. The author concluded that propranolol has an "excellent prophylactic effect" for children with frequent and severe attacks of migraine. ${ }^{18}$ A second study $(\mathrm{n}=39)$ failed to demonstrate preventive benefit at doses of $80-120 \mathrm{mg} /$ day and, in fact, significantly increased the average duration of headache in the propranolol group. ${ }^{19}$ A third trial compared propranolol at a dose of $3 \mathrm{mg} / \mathrm{kg} /$ day versus self-hypnosis and found no benefit from propranolol but significant improvement with hypnotherapy. ${ }^{20}$

Propranolol may be used on a single daily dose ("LA") form or on a bid or tid schedule. No comparative data exists. The starting dose is $1-2 \mathrm{mg} / \mathrm{kg} /$ day and slowly escalated to $3 \mathrm{mg} / \mathrm{kg} / \mathrm{day}$ as tolerated. Dosing adjustments can be made every $2-3$ weeks. 
Another beta-blocker, timolol was assessed in a randomized crossover trial with 8 weeks in each arm and a 4 week washout period between arms $(n=19)$. Headache attacks were reduced from 1.37 /week at baseline to 0.23 / week in the timolol group. In the placebo group, attacks were reduced from $1.06 /$ week baseline to $0.59 /$ week. The authors reported no significant beneficial effect from timolol. $^{21}$

The selective beta-blockers atenolol, metoprolol, and nadolol may be alternative choices, although controlled data to suggest any relative advantage is lacking.

Beta-blockers are contra-indicated in the presence of reactive airway disease, diabetes mellitus, orthostatic hypotension and certain cardiac disorders associated with bradyarrhthmias.

Special mention must be made about the use of betablockers in two other populations: athletes and patients with affective disorders, particularly depressions. Athletes may experience a lack of stamina and decreased performance. Those children with co-morbid affective disorders can experience deterioration of mood, even suicidal depression, with propranolol.

\section{Anti-depressants}

Anti-depressants have become a mainstay of migraine prophylaxis. While there is ample data to support the efficacy of anti-depressants for adult migraine, only two uncontrolled studies has been published for childhood migraine.

The first study included series of 192 children with headache, of whom $70 \%$ had migraine. The average age was 12 years and the patients had more than 3 headaches per month. They were treated in an open-label fashion with amitriptyline up to a dose of $1 \mathrm{mg} / \mathrm{kg} / \mathrm{day}$. Eighty four per cent reported an overall reduction in headache frequency and severity. Looking specifically at the migraine subset, there was a statistically significant reduction in headache frequency and severity, while the duration of headache attacks was unchanged when compared to initiation of the drug. Side effects were minimal. ${ }^{22}$

The second study was a retrospective review of the use of preventative agents for children and adolescents within one child neurology practice found that amitriptyline produced a "positive response rate" of $89 \%$ $(n=73)$. Positive response rate was defined as an overall decrease in headache frequency and intensity plus acceptability of the agent. Headache frequency was reduced from a mean baseline of 11 to 4.1 headaches per month. The principle side effect was mild sedation. ${ }^{17}$

The tricyclic anti-depressants amitriptyline, nortriptyline and desimpramine are widely employed and selection is generally a matter of personal preference and experience. There are no comparative data.

Amitriptyline is started as a single bedtime dose of 5-10 mg and slowly, every 4-6 weeks, titrated upward, as necessary, toward $25-50 \mathrm{mg}$. Sedation is the primary complication. Advantages of amitriptyline include low cost and its once-a-day schedule, which improves compliance. Some authorities recommend performance of ECG if doses in higher ranges are used.

Two other anti-depressant agents, trazadone, a triazolopyridine derivative and pizotifen, a serotonin-blocking agent (unavailable in the US), have been studied in controlled fashion, but both failed to demonstrate significant benefit. $^{23,24}$ The selective serotonin reuptake inhibitors (SSRI) have received attention, although, no controlled studies have been performed in children or adolescents. In those children and adolescents with co-morbid anxiety or depression, the SSRI's make be appropriate, however, recent FDA "black box" warning has emphasized suicide risks in adolescents taking this class of antidepressants. Giving careful consideration of these warnings, a morning dose of 10-20 mg of fluoxetine (Prozac) may be useful in this population with co-morbid affective disturbances.

Curiously, the efficacy of antidepressants is reported to be separate from their designed anti-depressant effect. However, as awareness of the common "co-morbidity" of affective disorders and migraine expands, the antidepressant properties may play an increasing and important role.

\section{Anti-epileptic agents}

Topiramate, valproate, levetiracetam and gabapentin, may have expanding roles for pediatric migraine. In light of the current views of the pathophysiology of migraine with a primary neuronal initiation and propagation through cortical excitation and, later, "spreading depression," anti-convulsants pose an intriguing, though yet incompletely defined role.

Topiratmate shows promise in adult migraine prevention. Adult trials were recently reported using topiramate at doses of $200 \mathrm{mg} /$ day (divided bid) and demonstrated a $50 \%$ reduction in headache frequency and severity. ${ }^{25}$ One retrospective study assessing the efficacy of topiramate for pediatric headache included 75 patients of whom 41 were available for follow-up visits. Daily doses of $1.4(+/-0.74) \mathrm{mg} / \mathrm{kg} /$ day were reached and headache frequency was reduced from $16.5(+/-10)$ headaches/ month to $11.6(+/-10)$ headaches/month $(\mathrm{p}<0.001)$. Mean headache severity, duration, and accompanying disability were also reduced. Side effects included cognitive changes $(12.5 \%)$, weight loss $(5.6 \%)$, and sensory symptoms $(2.8 \%)$. This population was predominantly children with chronic daily headache (15 headaches per month). ${ }^{26}$

Two abstracted retrospective reports of the use of topiramate for pediatric headache included 35 patients with migraine in whom there was a mean decrease in headache frequency of $70-77 \%$ at a mean dose of 
76-138 mg/day with mild adverse events including decreased appetite, sedation, paresthesias, and weight loss $(33 \%){ }^{27,28}$

A retrospective study of divalproex sodium (e.g.sodium valproate) for migraine prophylaxis in children, ages 7-16 years $(\mathrm{n}=42)$ at a dosing range of $15-45 \mathrm{mg} / \mathrm{kg} /$ day found $81 \%$ were successful in discontinuing all abortive (acute) medications. After 4 months of treatment, $75.8 \%$ of the patients reported a $50 \%$ reduction in headache frequency, $14.2 \%$ had a $75 \%$ reduction and $14.2 \%$ achieved a headache-free status. Side effects included GI upset weight gain, somnolence dizziness and tremor, similar to those experienced by patients with epilepsy. ${ }^{29}$

A second study using sodium valproate included children ages 9-17 years $(n=10)$ who were treated in an open label fashion with doses between 500-1000 mg. Both headache severity and frequency were reduced. Mean severity at baseline using a visual analog scale was reduced from 6.8 to 0.7 at the end of treatment $(p=0)$. Mean headache attacks per month were reduced from $6 /$ month to $0.7 /$ month and mean duration of headache attack was reduced from 5.5 hours to 1.1 hours following treatment. Side effects included dizziness, drowsiness, and increased appetite, but no serious side effects were noted in this small study. The authors conclude that sodium valproate is safe and effective for migraine prophylaxis in children. ${ }^{30}$

The doses used for valproate, as shown in table 1, are lower than those used for seizure control. A schedule of 10 $\mathrm{mg} / \mathrm{kg} /$ day or $250 \mathrm{mg}$ po bid or a single bedtime dose of the extended release preparation $(250 \mathrm{mg}, 500 \mathrm{mg})$, is a reasonable starting point. A similar monitoring schedule to that used for patients taking valproate for epilepsy applies with periodic measurements of blood counts, especially platelets, liver chemistries and amylase.

The acceptability of valproate in adolescent females warrants special mention in view of the appetite stimulation and risk of ovarian dysfunction (e.g. polycystic ovary).

Levetiracetam at doses of $125-250 \mathrm{mg}$ twice daily was assessed in a retrospective fashion including 19 patients (mean age 12 years) treated for a mean duration of 4.1 months. The average frequency of headache attacks before treatment was 6.3/month and after treatment, fell to $1.7 /$ month $(\mathrm{p}<0.0001)$. A striking $52 \%$ of patients experienced "elimination" of migraine attacks during treatment. No side effects were reported in $82.4 \%$ but $10.5 \%$ discontinued treatment because of side effects including somnolence, dizziness, and irritability. ${ }^{31}$

One retrospective study using gabapentin in children $(\mathrm{n}=18)$ at doses of $15 \mathrm{mg} / \mathrm{kg}$ found that over $80 \%$ of patients experienced a more than $50 \%$ reduction in headache frequency and severity. ${ }^{32}$ Perhaps the most desirable feature of gabapentin is the low incidence of side effects.
Clearly, more studies need in children to assess their efficacy and tolerability of anti-epileptic agents for migraine prevention.

\section{Calcium channel blockers}

Calcium channel blockers are thought to exert their effects through selective inhibition of vasoactive substances on cerebrovascular smooth muscle.

Nimodipine (10-20 mg tid) was studied in a single controlled, crossover trial including children ages 7-18 years $(n=37)$, but the results were inconsistent between the two treatment phases. During the first treatment period, there was no difference between active and placebo. Headache frequency per month fell from 3.3 to 2.8 in the active group and from 3.0 to 2.5 in the placebo group $(\mathrm{n}=\mathrm{NS})$. During the second treatment phase, there was a significant reduction in headache frequency in the nimodipine group, but there was no effect on headache duration. Side effects were limited to mild abdominal discomfort in $>1 \%{ }^{33}$

Although not available in the U.S., flunarizine, is a calcium channel blocker that has been evaluated in several well controlled trials. Two double-blind, placebocontrolled trials using $5 \mathrm{mg}$ bedtime doses of flunarizine $(n=105)$ and demonstrated significant reduction in headache frequency in both studies, one also showing decreased headache duration. ${ }^{34,35}$ In this first trial, the number of headaches was reduced from a baseline of 8.66 over 3 months to 2.95 attacks during treatment. Of patients taking flunarizine, $76 \%$ noted a $50 \%$ improvement, whereas only $19 \%$ taking placebo had $50 \%$ improvement 934). Another open-label trial of 13 patients showed decreased headache frequency. ${ }^{36}$ Other than sedation $(9.5 \%)$ and weight gain $(22.2 \%)$, side effects were minimal.

\section{Non-steroidal anti-inflammatory agents}

Naproxen sodium has been shown to be effective in adolescent migraine in one small series using a double blind, placebo-controlled crossover design. Sixty percent of the patients experienced a reduction in headache frequency and severity with naproxen $250 \mathrm{mg}$ bid whereas only $40 \%$ responded favorably to placebo. The rate limiting effect is gastrointestinal discomfort. ${ }^{37}$ For that reason, use should be limited to about 2 months duration.

Summary of preventive agents. Based upon recent review of the medical literature, the calcium channel blocker flunarizine is the only agent that has been studied in rigorous controlled trials and found to be effective. ${ }^{1,38}$ Flunarizine, however, is unavailable in the United States. There is uncontrolled data to suggest a beneficial effect with the antihistamine cyproheptadine, the antidepressant amitriptyline, the non-steroidal anti-inflammatory agent naproxen, and the anticonvulsants agent topiramate, valproic acid, and gabapentin. There is conflicting controlled evidence regarding propranolol and trazadone. 
Clonidine, pizotifen, nimodipine, and timolol were not shown to be more effective than placebo. The Cochrane Database (www.cochrane.org) concludes with the statement that there is a "clear and urgent need" for methodologically sound randomized controlled trials for the use of prophylactic drugs in pediatric migraine.

\section{SUMMARY}

The management of pediatric migraine requires a balance of bio-behavioral measures coupled with agents for acute treatment and, if needed, daily preventive medicines. A recent American Academy of Neurology practice parameter has critically reviewed the limited data regarding the efficacy and safety of medicines for the acute and preventive therapy of pediatric migraine (www.aan.org).

The first step is to establish the headache frequency and degree to which the migraines impact upon lifestyle and performance. The next step is to institute the nonpharmacological measures such as regulation of sleep (improved sleep hygiene), moderation of caffeine, regular exercise, and identification of provocative influences (e.g. stress, foods, social pressures). A wide variety of therapeutic options exist for patients whose migraine headaches occur with sufficient frequency and severity to produce a functional impairment.

The most rigorously studied agents for the acute treatment of migraine are ibuprofen, acetaminophen, and sumatriptan and zolmitriptan nasal spray, all of which have shown safety and efficacy in controlled trials.

Daily preventative drug therapies are warranted in about $20-30 \%$ of young migraine sufferers. The particular drug selected for the individual patient requires an appreciation of co-morbidities such as affective or anxiety disorders, co-existent medical conditions such as asthma or diabetes, and acceptability of potential toxicities such as weight gain, sedation, or tremor. A diverse group of anti-depressants, anti-hypertensives, anti-convulsants are available in table 6. Flunarezine (not available in the U.S.) is the most efficacious agent, but encouraging data is emerging regarding the use of several anti-epileptic agents such as topiramate, disodium valproate, levateracetam, as well as the antihistamine cyproheptadine and the anti-depressant amitriptyline.

\section{REFERENCES}

1. Lewis D., Ashwal S., Hershey A., et al. Practice Parameter: Pharmacological treatment of migraine headache in children and adolescents. Neurology 63:2215-2224, 2004.

2. Valquist B. Migraine in children. Internat Arch Allergy 7:348355, 1955.

3. Prensky AL, Sommer D. Diagnosis and treatment of migraine in children. Neurology 29:506-510, 1979.

4. Oleson J. The international classification of headache disorders. Cephalalgia 24(suppl. 1):1-160, 2004.
5. Silberstein SD. Practice Parameter: Evidence-based guidelines for migraine headache (an evidence-based review). Neurology 55: 754-762, 2000.

6. Powers S, Patton S, Hommell, K, Hershey A. Quality of life in paediatric migraine: characterization of age-related effects using PedsQL 4.0. Cephalalgia 24:120-127, 2004.

7. Hamalainen M., Hoppu K., Valkeila E., et al. Ibuprofen or acetaminophen for the acute treatment of migraine in children : a double-blind, randomized, placebo-controlled, crossover study. Neurology 48:102-107, 1997.

8. Lewis D., Kellstein D., Burke B, et al. Children's ibuprofen suspension for the acute treatment of migraine headache. Headache 42:780-786, 2002.

9. Hamalainen M. Sumatriptan for migraine attacks in children: A randomized placebo-controlled study. Do children with migraine respond to oral sumatriptan differently that adults? Neurology 48:1100-1103, 1997.

10. Winner P, Prensky A, Linder S, et al. Adolescent migraine: efficacy and safety of sumatriptan tablets. Journal of Neurological Sciences 150(suppl):S172, 1997.

11. Winner P, Rothner AD, Saper J, et al. Sumatriptan nasal spray in the treatment of acute migraine in adolescent. Pediatrics 106:989997, 2000.

12. Winner P, Rothner A, Webster C, Ames M. Overall efficacy of Sumatriptan Nasal Spray in Adolescent Migraineurs: Pooled Results from US Placebo-Controlled Trials. Headache 44:465, 2004.

13. Linder SL. Subcutaneous Sumatriptan in the Clinical Setting: The first 50 consecutive patients with acute migraine in the pediatric neurology office practice. Headache 36:419-422, 1996.

14. Winner P, Lewis D, Visser WH, et al. Rizatriptan $5 \mathrm{mg}$ for the acute treatment of migraine in adolescents: a randomized, doubleblind placebo-controlled study. Headache 42:49-55, 2002.

15. Linder SL, Dowson AJ. Zolmitriptan provides effective migraine relief in ado-lescents. Int J Clin Prac 54:466-469, 2000.

16. Lewis D, Winner P, Hershey A, et al. Zolmitriptan $5 \mathrm{mg}$ nasal spray is effective and well-tolerated in the acute treatment of adolescent migraine. European J of Neuro 12(Suppl 12):206, 2005.

17. Lewis D, Diamond S, Scott D, et al. Prophylactic treatment of pediatric migraine. Headache 44:230-237, 2004.

18. Ludvigsson J. Propranolol used in prophylaxis of migraine in children. Acta Neurologica 50:109-115, 1974.

19. Forsythe WI, Gillies D, Sills MA. Propranolol (Inderal) in the treatment of childhood migraine. Dev Med Child Neuro 26:737$741,1984$.

20. Olness K, MacDonald JT, Uden DL. Comparison of Self-Hypnosis and Propranolol in the Treatment of Juvenile Classic Migraine. Pediatrics 79:593-597, 1987.

21. Noronha MJ. Double-blind randomized cross-over trial of timolol in migraine prophylaxis in children. Cephalalgia 5(Suppl. 3):174175, 1985.

22. Hershey AD, Powers SW, Bentti AL and deGrauw TJ. Effectiveness of amitriptyline in the prophylactic management of childhood headaches. Headache 40:539-549, 2000.

23. Battistella P, Ruffilla R, Baldin L, et al. Trazodone prophylaxis of childhood migraine: A double blind placebo controlled cross-over study. Giornale di Neuropsichiatria Dell 'Eta Evolutiva 13:179_ 186, 1993.

24. Gillies D, Sills M, Forsythe I. Pizotifen (Sanomigran) in childhood migraine. A double-blind placebo controlled trial. 25:32-35, 1986.

25. Storey JR, Calder CS, Hart DE, Potter DL. Topiramate in migraine prevention: A double-blind, placebo-controlled study. Headache 41:968-975, 2001

26. Hershey AD, Powers SW, Vockell AL, et al. Effectiveness of topiramate in the prevention of childhood headache. Headache 42:810-818, 2002.

27. Younkin DP. Topiramate in the treatment of pediatric migraine Headache 42:456, 2002.

28. Ferreia J, Garcia N, Pedreira L. A case series of topiramate in pediatric and adolescent migraine prophylaxis. Headache 42:453, 2002.

29. Caruso JM, Brown WD, Exil G, Gascon GG. The efficacy of divalproex sodium in the prophylactic treatment of children with migraine. Headache 40:672-676, 2000. 
30. Serdaroglu G, Erhan E, Tekgul, et al. Sodium valproate prophylaxis in childhood migraine. Headache 42:819-822, 2002.

31. Miller GS. Efficacy and safety of levetiracetam in pediatric migraine. Headache 44:238-243, 2004.

32. Belman AL, Milazo M, Savatic M. Gabapentin for Migraine Prophylaxis in Children. Annals of Neurology 50(Suppl 1):S109, 2001.

33. Battistella PA, Ruffilli R, Moro R, Fabiani M, Bertoli S, Antolini A, Zacchello F. A placebo-controlled crossover trial of nimodipine in pediatric migraine. Headache 30:264-268, 1990.

34. Sorge F, Marano E. Flunarizine v. placebo in childhood migraine. A double-blind study. Cephalalgia 5(Suppl. 2):145-148, 1985.
35. Sorge F, DeSimone R, Marano E, et al. Flunarizine in prophylaxis of childhood migraine. A double-blind, placebo-controlled crossover study. Cephalalgia 8:1-6, 1988 .

36. Guidetti V, Moscato D, Ottaviano S, et al. Flunarizine and migraine in childhood an evaluation of endocrine function. Cephalalgia 7:263-266, 1987.

37. Lewis DW, Middlebrook MT, Deline C. Naproxen Sodium for Chemoprophylaxis of Adolescent Migraine. Annals of Neurology 36:542, 1994.

38. Victor S, Ryan S. Drugs for preventing migraine headaches in children. Cochrane Database System Reviews 4:CD 002761, 2003. 